\title{
Characterization of tree shrew (Tupaia belangeri) interleukin-6 and its expression pattern in response to exogenous challenge
}

\author{
YUANYUAN HAN $^{1}$, XIAOMEI SUN ${ }^{1}$, DEXUAN KUANG $^{1}$, PINFEN TONG $^{1}$, CAIXIA LU $^{1}$, WENGUANG WANG ${ }^{1}$, \\ $\mathrm{NA} \mathrm{LI}^{1}$, YANG CHEN ${ }^{2}$, XIAOPING WANG ${ }^{3}$, JIEJIE DAI $^{1}$ and HUATANG ZHANG ${ }^{4}$ \\ ${ }^{1}$ Center of Tree Shrew Germplasm Resources, Institute of Medical Biology, The Chinese Academy of Medical Sciences \\ and Peking Union Medical College, Yunnan Key Laboratory of Vaccine Research and Development on Severe Infectious Diseases, \\ Kunming, Yunnan 650118; ${ }^{2}$ MOE Key Laboratory of Bioinformatics and Bioinformatics Division, Center for Synthetic \\ and System Biology, TNLIST/Department of Automation, Tsinghua University, Beijing 100084; ${ }^{3}$ State Key Laboratory \\ for Conservation and Utilization of Bioresources in Yunnan, Yunnan University, Kunming, Yunnan 650091; \\ ${ }^{4}$ Chongqing Research Center of Biomedicine and Medical Equipment, Chongqing \\ Academy of Science and Technology, Chongqing 401123, P.R. China
}

Received March 24, 2017; Accepted September 19, 2017

DOI: $10.3892 /$ ijmm.2017.3168

\begin{abstract}
Tree shrews, one of the closest relatives of primates, have attracted increasing attention as a model of human diseases, particularly for viral infections. As the first line of defense against microbial pathogens, the innate immune system is crucial in tree shrews. Interleukin-6 (IL-6) is important in the pathophysiology of infection, inflammation and cancer, where it promotes disease development or sustains immune reactions. The present study aimed to obtain further insight into the tree shrew IL-6 (tsIL-6) system, and the function of tsIL- 6 in the antiviral and antibacterial response. In the present study, the mRNA and genomic sequence of the tsIL-6 gene were characterized, and the tissue distribution and expression profile of this gene were analyzed in response to lipopolysaccharide (LPS) and polyinosinic:polycytidylic acid (poly I:C) treatment. The full-length tsIL-6 mRNA consisted of $1,152 \mathrm{bp}$ with an open reading frame of $627 \mathrm{bp}$ encoding
\end{abstract}

Correspondence to: Professor Jiejie Dai, Center of Tree Shrew Germplasm Resources, Institute of Medical Biology, The Chinese Academy of Medical Sciences and Peking Union Medical College, Yunnan Key Laboratory of Vaccine Research and Development on Severe Infectious Diseases, 935 Jiaoling Road, Kunming, Yunnan 650118, P.R. China

E-mail:djj@imbcams.com.cn

Professor Huatang Zhang, Chongqing Research Center of Biomedicine and Medical Equipment, Chongqing Academy of Science and Technology, 2 Yangliu Road, Huangshan Avenue, New North Zone, Chongqing 401123, P.R. China

E-mail: zht@cast.gov.cn

Key words: tree shrew, interleukin-6, gene structure, gene function, expression pattern, immune challenge
208 amino acids, a 5'-untranslated region (UTR) of $62 \mathrm{bp}$, and a 3'-UTR of $436 \mathrm{bp}$. The genome sequence of the tsIL-6 gene was 5,265 bp in length, comprising of five exons and four introns. The predicted tsIL-6 protein contained a 25 -aminoacid-long signal peptide and a conserved IL-6 domain. Phylogenetic analysis based on the coding sequences revealed that tsIL- 6 was closely related to IL-6 in humans. Residues crucial for receptor binding were completely conserved in the tree shrew protein. Reverse transcription-polymerase chain reaction analysis revealed that tsIL-6 mRNA was expressed in all examined tissues of healthy tree shrews, with high levels in the muscle and spleen. Following poly I:C challenge, the expression levels of tsIL-6 were upregulated in four tissues associated with immune system, the liver, spleen, kidney and intestine. Taken together, the molecular and bioinformatics analyses based on the IL-6 sequence revealed that the tree shrew has a close phylogenetic association with humans. These results provide insight for future investigations on the structure and function of tsIL-6.

\section{Introduction}

The tree shrew (Tupaia belangeri) is widely distributed in South China and is in the order Scandentia. Based on several characteristics, including small body size, low cost, easy maintenance, short reproductive cycle, high brain-to-body mass ratio and, in particular, its close relation to primates, the tree shrew is being increasingly used as a valuable animal model and as an alternative animal model to rodents and non-human primates in biomedical research, including use as a model in investigations of viral hepatitis infection (1), depression (2), myopia $(3,4)$, tumors $(5)$, aging and learning behaviors $(6)$.

Interleukins (ILs) are the largest group of cytokines and are important in the innate and acquired immune responses of the host. Among them, IL-6 is vital in immune responses, including hematopoiesis, the acute phase of disease processes, immune cell differentiation and energy transduction, through 
several complex immune signaling pathways (7). Although IL-6 has a protective role in various bacterial and viral infections, the same activities can maintain chronic inflammation in conditions, including arthritis and other autoimmune diseases. Therefore, it is important to define the sequence features of IL-6 and obtain further insight into the function of tsIL-6 in the antiviral and antibacterial response to assist in investigations using tree shrews for models associated with IL-6.

To date, >89 IL-6 genes have been identified in mammals, and analyses of the homology of IL-6 among several species have been reported (8-12). Additionally, a number of important immune factors and important molecules in the tree shrew have been investigated in previous studies (13-15). However, as an important cytokine associated with viral infection, the genomic and mRNA sequence of tsIL- 6 has not been predicted (16) and associated investigations of expression have not been reported for the tree shrew, restricting its use in the modeling of human disease.

As the tree shrew is considered to be evolutionarily closer to humans, than rodents, they may serve as a superior animal model to investigate human disease mechanisms. The present study aimed to elucidate the structural characteristics of tsIL-6 and its expression pattern in response to exogenous challenge, in order to provide fundamental information on the gene structure and phylogeny of the tsIL- 6 gene. In addition, the tissue-specific distribution of IL-6 was examined in 14 tissue specimens, and its expression profiles following lipopolysaccharide (LPS) and polyinosinic:polycytidylic acid (poly I:C) stimulation in four tissues associated with the immune system were examined using reverse transcription-quantitative polymerase chain reaction (RT-qPCR) analysis.

\section{Materials and methods}

Laboratory animals and exogenous challenge. Healthy 1-year-old, male tree shrews (Tupaia belangeri; length, $17.89 \pm 0.49 \mathrm{~cm}$; weight, $149.80 \pm 15.26 \mathrm{~cm})$ were provided by and maintained at the Center of Tree Shrew Germplasm Resources, Institute of Medical Biology, The Chinese Academy of Medical Science and Peking Union Medical College (Kunming, China). The production certification number was SCXK (Dian) K2013-0001. The institutional Animal Care and Welfare Committee of the Institute of Medical Biology, Chinese Academy of Medical Sciences and Peking Union Medical College approved the present study, and all the procedures were performed according to ethical standards and practices.

Immune stimulation was performed by intraperitoneal injection of $500 \mu \mathrm{l}$ LPS $(1.5 \mathrm{mg} / \mathrm{ml}$; cat. no. L2630) or $500 \mu 1$ Poly I:C (1.5 mg/ml; cat. no. P9582) (both from Sigma-Aldrich, St. Louis, MO, USA) in phosphate-buffered saline (PBS; pH 7.4; HyClone Media, Logan, UT, USA), respectively $(11,12)$. Tree shrews injected with $500 \mu$ l PBS were used as controls. Following injection, all tree shrews were placed back into their cages and housed individually. All animals were housed in the same room under a $12 \mathrm{~h}$ light/12 h dark cycle (lights on at 08:00; 150 lux) with $60 \pm 10 \%$ relative humidity and an ambient temperature of $25 \pm 2^{\circ} \mathrm{C}$. The tree shrews had ad libitum access to water and food. At $0,3,6,12,24,48$ and $72 \mathrm{~h}$ post-injection, two tree shrews in each group were sacrificed and four samples of tissues associated with the immune system, the liver, spleen, kidney and intestine, were obtained for total RNA extraction.

RNA isolation, cDNA synthesis and cloning, and sequencing of tsIL-6 mRNA. Total RNA was extracted from $100 \mathrm{mg}$ of tree shrew splenic tissue with TRIzol reagent (Invitrogen; Thermo Fisher Scientific, Inc., Waltham, MA, USA) according to the manufacturer's protocol, and incubated in RNase-free DNase I (Takara Biotechnology Co., Ltd., Dalian, China) for 20-30 min at $37^{\circ} \mathrm{C}$ to remove residual genomic DNA. First-strand cDNA was synthesized from the total RNA using M-MLV reverse transcriptase (Fermentas; Thermo Fisher Scientific, Inc., St. Leon-Rot, Germany) with the 5'-rapid amplification of mRNA ends (RACE) RT primer and oligo(dT) $)_{18}$.

Based on the published mRNA sequence of tsIL-6 (16), a pair of primers, EXONs 1-5, targeting the two ends of tsIL-6 was designed (Table I). The 5' and $3^{\prime}$ ends of tsIL-6 were amplified using the RACE approach (17). The 5' end RACE PCR reaction was performed using the 5'-RACE 1 adapter forward primer and a gene-specific 5'-RACE 1 reverse primer, followed by nested PCR with a 5'-RACE 2 adapter forward primer and a gene-specific 5'-RACE 2 reverse primer. The $3^{\prime}$ end RACE PCR reaction was performed using the genespecific 3'-RACE 1 forward primer and 3'-RACE 1 adapter reverse primer, followed by nested PCR with a gene-specific 3'-RACE 2 forward primer and the 3'-RACE 2 adapter reverse primer. Approximately $1 \mu \mathrm{l}$ cDNA synthesized from total RNA from tissues was used as the template. The reaction was performed in a volume of $20 \mu \mathrm{l}$ containing $0.4 \mu \mathrm{M}$ of each primer, $200 \mu \mathrm{M}$ dNTPs, 1 unit of LA TaqDNA polymerase (Takara Biotechnology Co., Ltd.) and $2 \mu 110 \mathrm{X}$ buffer. We used the following PCR conditions: one denaturation cycle at $95^{\circ} \mathrm{C}$ for $2 \mathrm{~min}, 35 \mathrm{cycles}$ of $94^{\circ} \mathrm{C}$ for $30 \mathrm{sec}, 57^{\circ} \mathrm{C}$ for $30 \mathrm{sec}$ and $72^{\circ} \mathrm{C}$ for $30 \mathrm{sec}$, followed by one cycle of $72^{\circ} \mathrm{C}$ for $10 \mathrm{~min}$.

All PCR products were gel-purified, cloned into the pMD19-T vector (Takara Biotechnology Co., Ltd.), and commercially sequenced by Sangon Biotech Co., Ltd. (Shanghai, China). The sequences of overlapping clones were assembled to construct the complete tsIL-6 mRNA sequence.

DNA extraction and cloning of tsIL-6 genomic DNA. Total DNA was isolated from the splenic tissues using a TIANamp Marine Animals DNA kit (cat. no. DP324; Tiangen Biotech Co., Ltd., Beijing, China) according to the manufacturer's protocol. Targeting the conserved regions of mammalian IL-6 genomic sequences, five pairs of primers (genome 1-5) were designed to amplify the genome of tsIL-6 (Table I). All PCR products were gel-purified, cloned into the pMD19-T vector (Takara Biotechnology Co., Ltd.), and sequenced by Sangon Biotech Co., Ltd. The sequences of overlapping clones were assembled to construct the complete tsIL-6 genomic gene sequence. The tsIL- 6 genomic and mRNA sequences were then aligned using DNAMAN software (version 8.0; Lynno Biosoft, San Ramon, CA, USA) to verify the intron/exon boundaries.

Amino acid sequences and phylogenetic analysis. The promoter sequence was analyzed using the JASPAR database (http://jaspar.genereg.net/cgi-bin/jaspar_db.pl). Homology sequence searching was performed using the Basic Local 
Table I. Primer pairs used in polymerase chain reactions.

\begin{tabular}{lll}
\hline Primer pair & \multicolumn{1}{c}{ Forward primer (5'-3') } & \multicolumn{1}{c}{ Reverse primer (5'-3') } \\
\hline EXONS 1-5 & CCACAAGCACCTTCAGTCCAG & CAAAGGAAGGAATGTCCATGTCTA \\
5'-RACE 1 & GCCACGCGTCGACTAGTACGGGGGGGGGG & GCCAGTGCAACCCTGCACTTGTAAA \\
5'-RACE 2 & GGCCACGCGTCGACTAGTAC & TCTTCAGTTTTATCTGTGGAGGTGGGTCT \\
3'-RACE 1 & TCCCACCCCTGACCCAACTTCAAAT & GCTGTCAACGATACGCTACGTAAC \\
3'-RACE 2 & TCAAGAGGAGTGGCTAAAGAAGGTGACAGT & GCTACGTAACGGCATGACAGTG \\
Genome 1 & ACGCTTATACTTTTAGTTCTTCATGGA & CAGCTATGCCAGGCAGTGTT \\
Genome 2 & TGTGTCTGAGTTTTAAGCTGCCA & GGCAGAATGAAGCACATCCAA \\
Genome 3 & CTGGAGGAGAAGAAATTGTGGAG & TCCTTCTAGCTCTTTCTTAGGCAA \\
Genome 4 & CAACCCAGAGGGACCAATTT & CAGGCCATTCATTTCCTTCT \\
Genome 5 & AGTGCCAAAGTCCAAGGGTC & TCTGTCCTTGAGCCCACCA \\
GAPDH & CCATCACCATCTTCCAGGAGCGAG & CAAAGGTGGAGGAGTGGGTGTCG \\
IL-6 qPCR & CCTGACCCAACTTCAAATGC & CACACTACATTAGCCGAGAAGC \\
GAPDH & CCATCACCATCTTCCAGGAGCGAG & CAAAGGTGGAGGAGTGGGTGTCG \\
\hline
\end{tabular}

RACE, rapid amplification of mRNA ends; IL-6, interleukin-6; qPCR, quantitative polymerase chain reaction; GAPDH, glyceraldehyde 3-phosphate dehydrogenase.

Alignment Search Tool (http://www.ncbi.nlm.nih.gov/blast). Deducedaminoacidsequencecomparisonswereperformedusing the Expert Protein Analysis System (http://www.expasy.org/). The signal peptide and conserved domains were predicted using the Simple Modular Architecture Reach Tool (http://smart. embl-heidelberg.de/). Glycosylation sites were predicted using NetOGlyc 3.1 (http://www.cbs.dtu.dk/services/NetOGlyc). Phosphorylation sites were predicted using the NetPhos 2.0 server (http://www.cbs.dtu.dk/services/NetPhos/).

Multiple alignments of the putative amino acid sequences of tsIL-6 with IL- 6 from other related species were performed using MUltiple Sequence Comparison by Log-Expectation (18). Phylogenetic trees were obtained based on the IL-6 amino acid sequences (data in https://www.ncbi. nlm.nih.gov/nuccore/1051343575?report=fasta) by Bayesian inference (BI) using MrBayes software v.3.2.6 (http://mrbayes. sourceforge.net/) $(19,20)$ and by maximum likelihood (ML) using RAxML v.7.0.4 (https://sco.h-its.org/exelixis/web/software/raxml/) (21). In BI, two independent sets of Markov chain Monte Carlo algorithms were run, each with three heated and one unheated chain, for 1,000,000 generations. The trees were sampled every 1,000 generations. The first 2,500 trees were discarded as burn-in. In ML analyses, the 'rapid bootstrap' option was run from starting random seeds to generate 100 nonparametric bootstrap replicates and the GTR-GAMMA model, the other parameters were set as default. Cows were used as an outgroup in both analyses.

Modeling of tsIL-6. The spatial protein structure was predicted using the SWISS-MODEL workspace (http://www.expasy. ch/swissmod/SWISS-MODEL.html) and was illustrated using the PyMOL Molecular Graphics system version 1.7.2.1 (DeLano Scientific, San Carlos, CA, USA).

RT-qPCR of $t s I L-6$. The RT-qPCR reactions were performed using SG Fast qPCR Master mix (High Rox) in a Stepone Plus system (both from ABI; Thermo Fisher Scientific, Inc.). For each reaction, a volume of $20 \mu \mathrm{l}$ containing $0.4 \mu \mathrm{M}$ of each forward and reverse primer, $2 \mu \mathrm{l}$ of cDNA product, and $10 \mu \mathrm{l}$ of 2X SYBR-Green Premix Ex Taq II were used for the RT-qPCR reaction. The primer sequences are provided in Table I (IL-6 qPCR primer). The cycling profile consisted of an initial denaturation at $95^{\circ} \mathrm{C}$ for $3 \mathrm{~min}$, followed by 45 cycles of $95^{\circ} \mathrm{C}$ for $7 \mathrm{sec}, 57^{\circ} \mathrm{C}$ for $10 \mathrm{sec}$, and $72^{\circ} \mathrm{C}$ for $15 \mathrm{sec}$, followed by melt curve analysis. The tree shrew housekeeping gene glyceraldehyde 3-phosphate dehydrogenase (GAPDH) was used as the reference gene for normalization (22). The normalized mRNA expression information of tree shrew, human, mouse and rat from BioGPS (www.biogps.org) was used in order to compare IL-6 gene basal expression in different tissues of tree shrew, human, mouse and rat.

Statistical analysis. Data were analyzed using one-way analysis of variance and Student-Newman-Keuls test with SPSS software (version 15; SPSS, Inc., Chicago, IL, USA), The results are expressed as the mean \pm standard error of the mean. $\mathrm{P}<0.05$ was considered to indicate a statistically significant difference.

\section{Results}

Structural features of the tsIL-6 gene. The structural characteristics of the complete tsIL-6 mRNA are shown in Fig. 1. An mRNA fragment of $659 \mathrm{bp}$ was amplified using the degenerate primers, EXONs 1-5. A fragment of $226 \mathrm{bp}$ containing the initial start codon ATG was then amplified by 5 ' RACE, and a 553-bp fragment containing the poly(A) tail was obtained by 3' RACE. Alignment with the DNA sequence produced a 1,152-bp mRNA sequence with five putative open reading frames (ORFs). Therefore, the 1,152-bp sequence representing the full-length mRNA sequence of tsIL-6 was obtained (accession no. KX359391), which contained an ORF of $627 \mathrm{bp}$, a 62-bp 5'-untranslated region (UTR), a 463-bp 3'-UTR (Fig. 1), and six RNA instability motifs (ATTTA) in the 3'-UTR (23). 


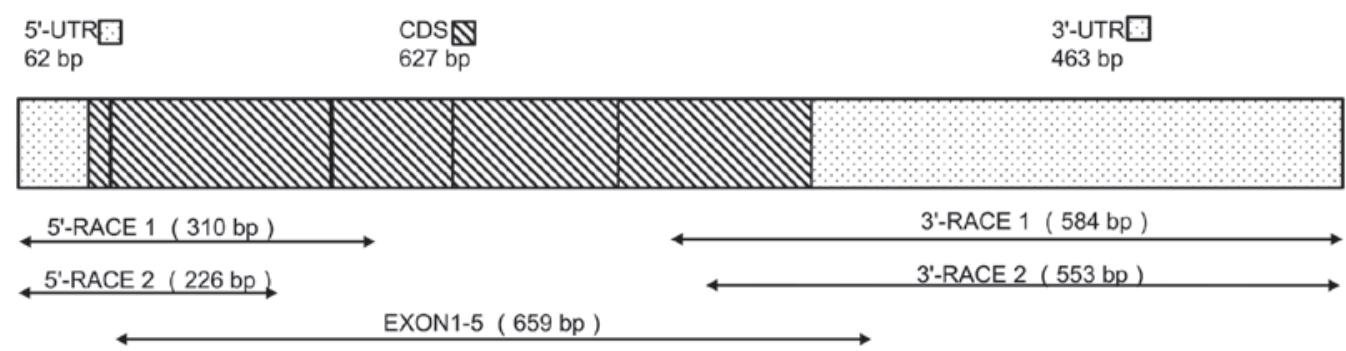

Figure 1. Schematic representation of tsIL-6 mRNA. The 5' and 3'-UTRs are indicated by dotted boxes. The coding region was divided into five exons, which are denoted by hatched boxes; the relative size of each box represents the exon length. The position of mRNA clones produced by each primer pair used for polymerase chain reaction is shown relative to the assembled mRNA sequence (GenBank accession KX359391). UTR, untranslated region; RACE, rapid amplification of mRNA ends; CDS, coding sequence; tsIL-6, tree shrew interleukin-6.

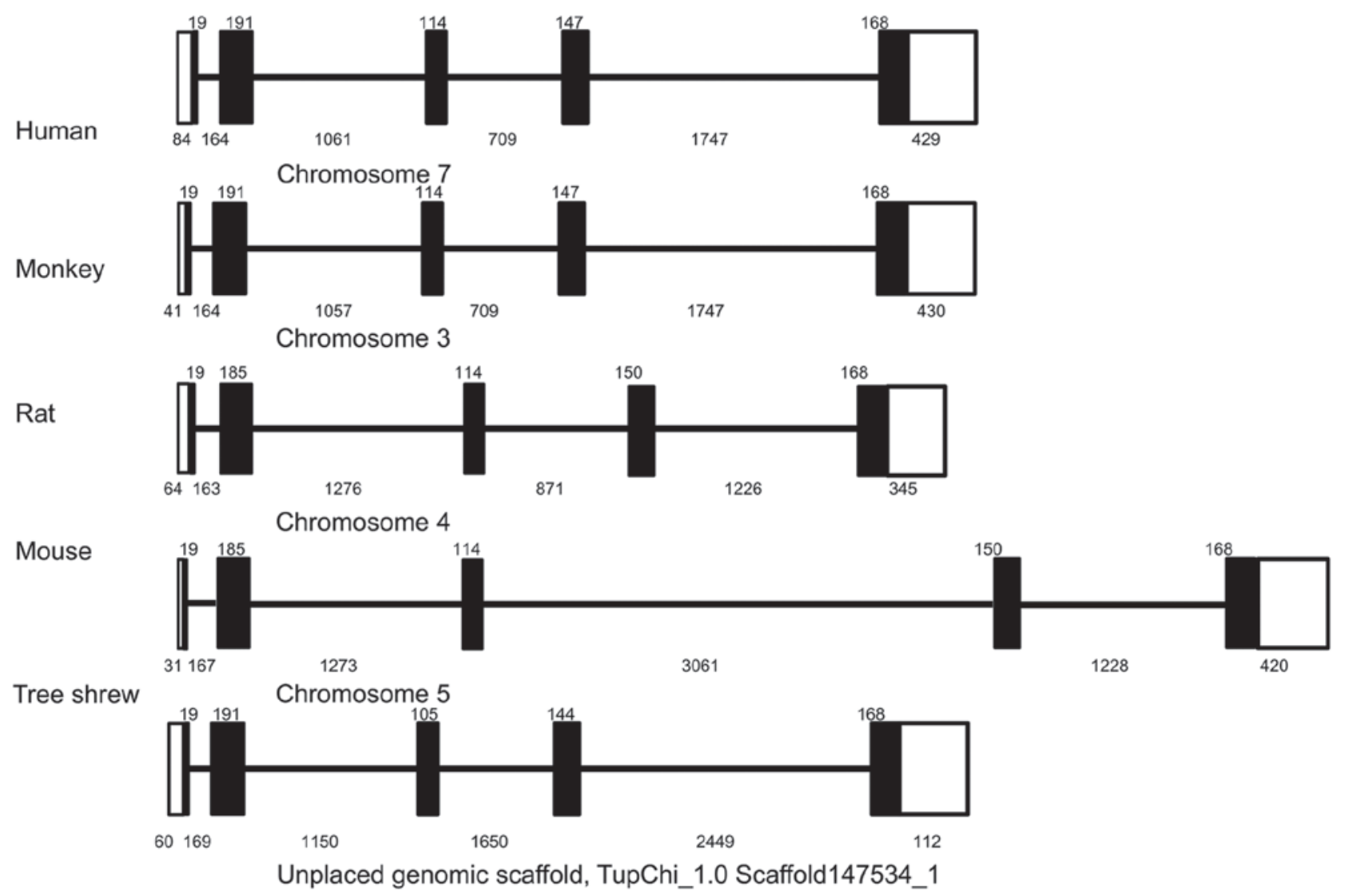

Figure 2. Diagram comparing the IL-6 gene. Diagram comparing the IL-6 gene from human (NC_000007.14), monkey (NC_027895.1), rat (NC_005103.4), mouse (NC_000071.6) and tree shrew (KX359391). Exons are represented by black boxes, promoters/terminators are represented by white boxes and lines indicate introns. The number of exons and promoter/terminator lengths are shown above the boxes, and the number of introns and their lengths are shown below the lines. The location of each IL- 6 on the chromosome is marked under the gene map for each species. IL-6, interleukin- 6 .

Using a similar strategy, the 5,265-bp genomic sequence of the tsIL- 6 gene was obtained and submitted to GenBank (accession no. KX359390). In contrast to the structure of the mRNA, the genomic sequence revealed that the tsIL-6 gene consisted of five exons and four introns (Fig. 2). All four introns had the typical structure of 'GU-AG', in which the first two nucleotides were 5 '-GU-3', and the last two nucleotides were 5'-AG-3'). There was a TATA box with the typical sequence of TATAATAAT between bases -548 and $-539 \mathrm{bp}$, and a CAAT box with a typical sequence of GGC/TCAATCT between bases -605 and $-596 \mathrm{bp}$. Analysis of the promoter region of tsIL-6 (between -400 and $+50 \mathrm{bp}$ from the transcription initiation site) for known conserved elements using JASPAR revealed several putative transcription factor binding regions and showed a high degree of conservation of the mammalian IL-6 gene. The highly conserved NFI, FOS, MAFG, CEBP, LBX1, DuxLhx8, Arid3a and REL sequences were identified, shown in Fig. 3A. The comparison of functional cis-regulatory elements from human and tsIL-6 promoter regions is shown in Fig. 3B (24).

Features of predicted amino acid sequences. Using the SMART program, the 627-bp ORF of the putative tsIL-6 was predicted to encode 208 amino acids, consisting of a signal peptide in the first 25 amino acids of the $\mathrm{N}$-terminus and an IL-6 domain between amino acid positions 57 and 206, with an E-value of 1.54e-56 (Fig. 4). The molecular weight and 


\begin{tabular}{|c|c|c|c|c|c|c|c|}
\hline \multirow[t]{15}{*}{ A } & \multirow[t]{2}{*}{-400} & \multirow{2}{*}{ GTAAAACGTC } & \multirow{2}{*}{ CTACGTGACC } & \multirow[t]{2}{*}{ AGCTTTCTTT } & CTCAAGA CAT & GCCAAAATGC & \multirow[t]{2}{*}{-351} \\
\hline & & & & & \multicolumn{2}{|c|}{$\mathrm{NFI}$} & \\
\hline & \multirow[t]{2}{*}{-350} & \multirow{2}{*}{$\frac{\text { TGAGTCACT }}{\text { Mafb /FOS }}$ A } & \multirow[t]{2}{*}{ AAAAAAATTT } & \multirow[t]{2}{*}{ TAAAAAAGAA } & \multirow[t]{2}{*}{ AGTAAAGGAA } & \multirow[t]{2}{*}{ GAGTGATCTT } & \multirow[t]{2}{*}{-301} \\
\hline & & & & & & & \\
\hline & -300 & GCTTCTTAGA & GATAGCCTCA & $\frac{\text { TT GATGAC }}{\text { MAFG }} T$ & AAGCTGCACT & TTTTTCCCCA & -251 \\
\hline & \multirow[t]{2}{*}{-250} & \multirow[t]{2}{*}{ TTTGTAATTC } & \multirow[t]{2}{*}{ GGTGATGCTA } & \multirow[t]{2}{*}{ AAGGACGTCA } & CATTACACAA & TCITAATAAG & \multirow[t]{2}{*}{-201} \\
\hline & & & & & HLF/CEBP & LBX1 & \\
\hline & \multirow[t]{2}{*}{-200} & GTTT CCAATC & AG CCCCACCC & \multirow[t]{2}{*}{ GTTCTGTCCC } & \multirow[t]{2}{*}{ САССTCCTAC } & \multirow[t]{2}{*}{ CСTCCAACAA } & \multirow[t]{2}{*}{-151} \\
\hline & & Dux/Lh & & & & & \\
\hline & \multirow[t]{2}{*}{-150} & AGATT TATCA & AA TG TGGGAT & TTCC CCATGA & \multirow[t]{2}{*}{ GTCTCAATAT } & \multirow[t]{2}{*}{ TAGAGTTTCA } & \multirow[t]{2}{*}{-101} \\
\hline & & & REL & & & & \\
\hline & \multirow[t]{2}{*}{-100} & \multirow{2}{*}{\multicolumn{2}{|c|}{ ACCTCCA $\frac{\text { ATA } \quad \text { AATA TGAGAC }}{\text { FOXL1 }}$}} & TGGGGA TGTC & \multirow[t]{2}{*}{ TGAGGTTCAT } & TCTGTCC TTG & -51 \\
\hline & & & & MZF1 & & & \\
\hline & -50 & AGCCC ACCAG & GAACGAAAGA & GCGCTCCATC & TGCCCTCCAG & AAACCCAGCT & -1 \\
\hline & & RHOXF1 & & & & & \\
\hline & + & ATG ACCTCCT & TGTCCACAAG & САCCTTCAGT & CCAGTTGCCT & TCTCCCTGG & 50 \\
\hline
\end{tabular}

Human IL-6

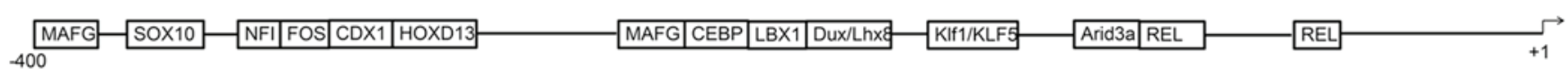

Tree shrew IL-6

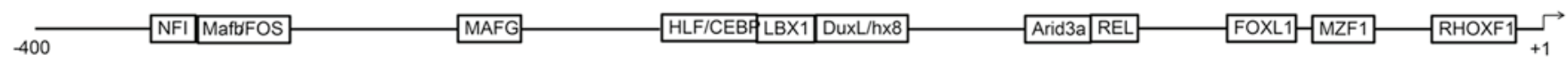

Figure 3. Cis-regulatory elements. (A) Prediction of functional cis-regulatory elements in the tsIL-6 promoter region (between -400 and +50 bp from the transcription initiation site. (B) Comparison of functional cis-regulatory elements from human and tree shrew IL-6 promoter regions. IL-6, interleukin-6.

isoelectric point were theoretically calculated as 23.46 and $7.97 \mathrm{kDa}$, respectively. All four conserved cysteine residues in mammalian IL-6 were observed at amino acid positions 72, 78, 98 and 108 (Fig. 4). The typical C-X(9)-C-X(6)-G-L$\mathrm{X}(2)-[\mathrm{FY}]-\mathrm{X}(3)-\mathrm{L}$ IL-6 family motif and conserved disulfide bridges were also identified in the tsIL-6 protein (Fig. 4). Nine positive positions were more likely to have O-GalNAc modifications, which were present at amino acid positions 2, 3, 5, 7, 8, 10, 158, 162 and 177 .

The alignment between human IL- 6 and tsIL- 6 revealed 111 identical amino acid residues. There were 97 amino acid residues in human IL-6, which were not found in the corresponding positions in the tree shrew. Among these, 35 amino acid residues were conserved and 43 amino acid residues were semi-conserved substitutions, and unlikely to affect protein structure or function. The remaining 19 residues were non-conserved substitutions. There were five non-conserved residues located close to the $\mathrm{N}$-terminus, at a position distant from the residues involved in receptor binding. There were 14 non-conserved residues located at sites of variable amino acid regions (labeled in Fig. 5). The structural and mutational analyses of human IL-6 identified residues 49-73, 108-130, 137-157, 169-180 and 184-210 as $\alpha$-helix A, B, C, D and E, respectively, and residues $72 \mathrm{C}-78 \mathrm{C}$ and $101 \mathrm{C}-111 \mathrm{C}$ as putative disulfide bond sites (Fig. 4). Similar $\alpha$-helical structure and disulfide bond sites were predicted in the corresponding sites in tsIL-6. The conserved (C-x(9)-C-x(6)-G-L-x(2)-[FY]-x(3)-L) peptide located at position $98-123$ consisted of a partial coil and a partial $\alpha$-helix B (Fig. 5).

Features of the three-dimensional structure. Analysis of the secondary structure of tsIL-6 revealed that the protein consisted of a bundle of four $\alpha$-helices linked by loops and an additional mini-helix (Fig. 5). There were three main IL-6 binding sites: Site I, II and III. Site I was responsible for IL-6-IL-6 receptor interactions. In the site II region, IL-6 interacted with gp130 in the trimer. Site III was the site of IL-6-gp130 interactions between trimers. Phe74, Gln175, Ser176, Arg179 and Arg182 in site I; Tyr31, Gly35, Ser118 and Val121 in site II; and Trp157 and Asp160 in site III were identified as important for binding based on mutagenesis investigation (Fig. 6A). The corresponding amino acids were Phe103, Asn103, Ser205, Arg203 and Arg206 in site I; Tyr59, Glu35, Leu146 and Met149 in site II; and Trp181 and Lys184 in site III. The surface charge appeared to be similar in corresponding site I, and less similar in sites II and III, suggesting potentially different proteinprotein interactions in human and tsIL-6 (Fig. 6B and C). The three-dimensional domain structures of human and tsIL- 6 were dimensionally conserved.

Phylogenetic relationship between the tree shrew and related species. The data comprised orthologs from five taxa, including representatives of primates (humans and macaques), Rodentia (mouse and rat) and the Cow outgroup. Phylogenetic trees were reconstructed using BI and ML methods. The BI and ML analyses supported the sister clades between tree shrew and primates with posterior probabilities $(\mathrm{PP}=0.68)$ and bootstrap support $(\mathrm{BP}=100)$ (Fig. 7).

Feature of expression profiles. To further determine the tsIL-6 mRNA expression distribution in the tree shrew, RT-qPCR was used to measure the mRNA expression levels of tsIL-6 in 14 tissue specimens from adult tree shrews. The highest expression levels were found in the muscle and the spleen, followed 


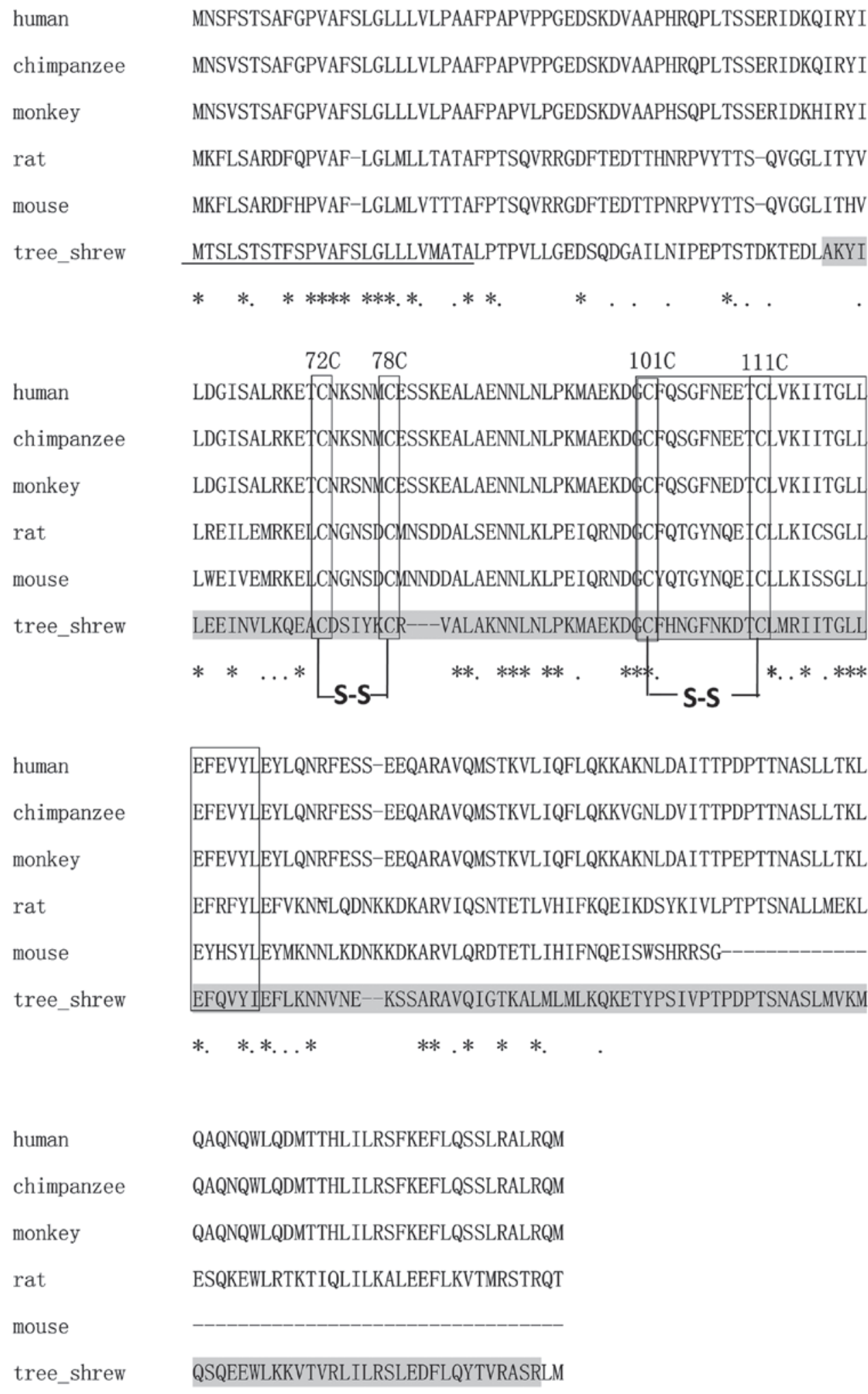

Figure 4. Multiple alignments of the deduced tree shrew IL-6 domain with known IL-6 domains from other common mammals. The predicted signal peptide is underlined. The IL-6 family signature, (C-x(9)-C-x(6)-G-L-x(2)-[FY]-x(3)-L), and the four conserved cysteine residues within boxes. '*' indicates conserved residues; '.' indicates less conserved residues. 'S-S' indicates the positions of conserved deduced disulfide bridges. tsIL-6, tree shrew interleukin-6.

by the testis, adipose tissue, spinal cord, adrenal gland, lung, intestine, heart, liver and kidney, whereas low expression was found in the cerebrum, cerebellum and hippocampus (Fig. 8). The present study further retrieved the normalized mRNA expression information of humans, mice and rats from BioGPS (www.biogps.org), in order to compare IL-6 gene basal expression in different tree shrew, human, mouse and rat tissues (Fig. 9). However, the data from the human splenic tissue, and the rat liver, lung, adipose, adrenal gland, spinal cord and testicular tissues were unavailable in BioGPS. Based on the relative abundance of mRNA expression of IL- 6 in human whole blood in BioGPS, it was hypothesized that the 


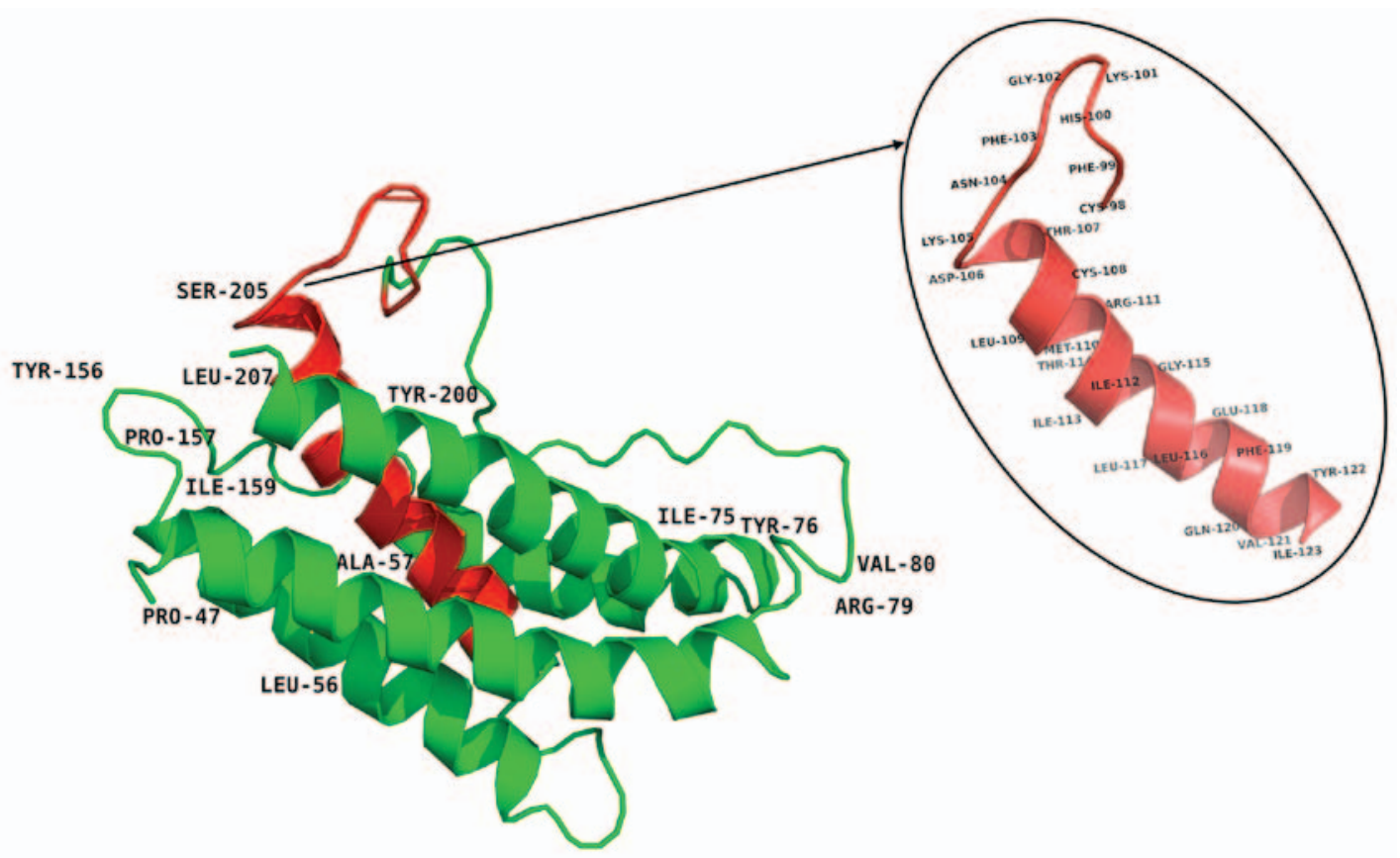

Figure 5. Comparison of amino acids between tsIL- 6 and human IL-6. The location of amino acid differences between the tree shrew and human IL-6 protein are indicated by labels. For each position in which the sequence differs, the label shows the three-letter amino acid code for the tsIL-6 and the position in the sequences. The conserved (C-X(9)-C-X(6)-G-L-X(2)-[FY]-X(3)-L) peptide sequence is shown as a red helix, and the details of the amino acid are magnified. tsIL-6, tree shrew interleukin-6.

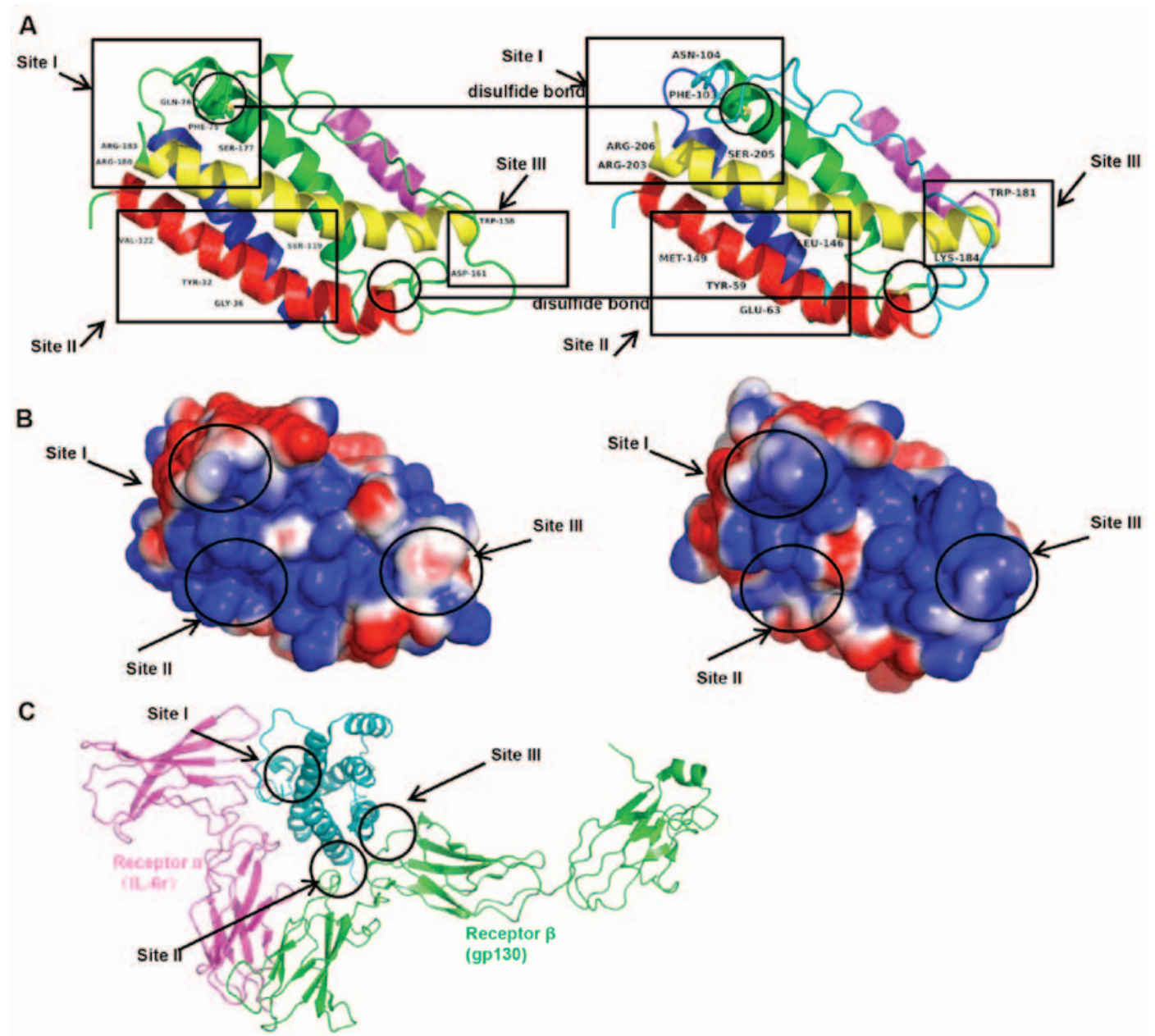

Figure 6. Modeling of the three-dimensional structures of the domains of human (left) and tree shrew (right) IL-6 chains. (A) Amino acids important for binding in sites I, II, III are shown within circles. (B) Surface charges in the domains of human and tsIL- 6 chains. Positive and negative surfaces are shown in blue and red, respectively $( \pm 5.0 \mathrm{kT} / \mathrm{e})$. (C) Receptor binding domains of human IL-6 chains. tsIL-6, tree shrew interleukin- 6 . 
Table II. Expression levels of tsIL-6 in 14 tissues.

\begin{tabular}{lcccccccccccccc}
\hline $\begin{array}{l}\text { Expression } \\
\text { level }\end{array}$ & $\begin{array}{c}\text { Cere- } \\
\text { brum }\end{array}$ & $\begin{array}{c}\text { Cere- } \\
\text { bellum }\end{array}$ & $\begin{array}{c}\text { Hippo- } \\
\text { campus }\end{array}$ & cord & Heart & Liver & Spleen Lung & Kidney Muscle & \multicolumn{4}{c}{$\begin{array}{c}\text { Adrenal } \\
\text { gland }\end{array}$} & Intestinal Testis Adipose \\
\hline Tree shrew 1 & 4.00 & 2.59 & 1.49 & 11.40 & 6.58 & 5.05 & 23.56 & 9.66 & 4.20 & 31.69 & 11.33 & 7.76 & 15.59 & 10.25 \\
Tree shrew 2 & 4.52 & 2.82 & 1.39 & 11.63 & 7.44 & 4.39 & 20.73 & 10.05 & 3.36 & 37.39 & 8.84 & 5.97 & 13.72 & 11.99 \\
Tree shrew 3 & 4.32 & 2.25 & 1.48 & 11.74 & 5.66 & 5.00 & 20.26 & 9.28 & 4.78 & 35.81 & 8.95 & 8.53 & 15.28 & 12.50 \\
Tree shrew 4 & 3.52 & 2.80 & 1.32 & 13.23 & 6.06 & 4.44 & 18.85 & 8.41 & 5.16 & 23.77 & 10.65 & 7.29 & 12.01 & 8.30 \\
Tree shrew 5 & 3.40 & 2.51 & 1.26 & 11.06 & 6.19 & 4.14 & 23.56 & 11.50 & 4.28 & 30.10 & 11.67 & 8.77 & 17.78 & 9.43 \\
Tree shrew 6 & 3.96 & 2.98 & 1.79 & 10.15 & 5.73 & 5.80 & 21.44 & 10.72 & 3.27 & 30.42 & 9.06 & 7.06 & 20.27 & 9.53 \\
\hline
\end{tabular}

The data were determined from 6 control adult tree shrews. tsIL- 6 , tree shrew interleukin- 6 .

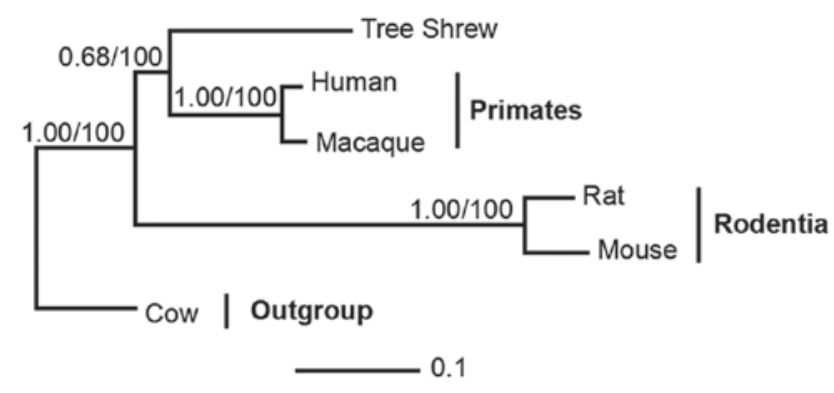

Figure 7. Evolutionary relationships of taxa. Phylogenetic trees obtained from Bayesian inference and maximum likelihood, based on the analysis of IL-6 amino acid sequences. The posterior probability and bootstrap score are shown on nodes. IL-6, interleukin-6.

expression level of human IL-6 was likely to be higher in splenic tissues than the other tissues, such as brain and liver, while the expression level of rat IL-6 was likely to be similar in all tissues. It appeared that the expression levels in humans and mice were similar, whereas the expression of IL-6 in the tree shrew muscle tissue was more variable and the highest basal mRNA expression was found here.

Expression of tsIL-6 in response to LPS and poly I:C challenge in vivo. The transcriptional changes of tsIL-6 in four immune system-associated tissues following injection with LPS and poly I:C are shown in Fig. 10. All tsIL-6 expression data is available in Tables II and III.

Following LPS challenge, tsIL-6 mRNA was significantly increased at $3 \mathrm{~h}$ in the liver $(\mathrm{P}<0.05)$, with the highest expression being 16.86-fold higher than that in the control, following which it reduced gradually. However, the significantly high expression levels were maintained to $48 \mathrm{~h}(\mathrm{P}<0.05)$. In addition, tsIL-6 transcription increased significantly at $3 \mathrm{~h}$ post-poly I:C injection, reaching a peak value of 39.23 -fold higher than that in the control $(\mathrm{P}<0.05)$, following which it showed gradual recovery and returned to control levels from 48-72 h post-injection (Fig. 10A).

Following stimulation with LPS, the mRNA expression of tsIL-6 increased significantly between 3 and $6 \mathrm{~h}$ post-injection in the spleen $(\mathrm{P}<0.05)$, with the highest value 23.7-fold higher than that of the control at $3 \mathrm{~h}$. The expression subsequently returned to the control level at $12 \mathrm{~h}$ post-stimulation. The mRNA expression of tsIL-6 increased gradually between 3 and $12 \mathrm{~h}$

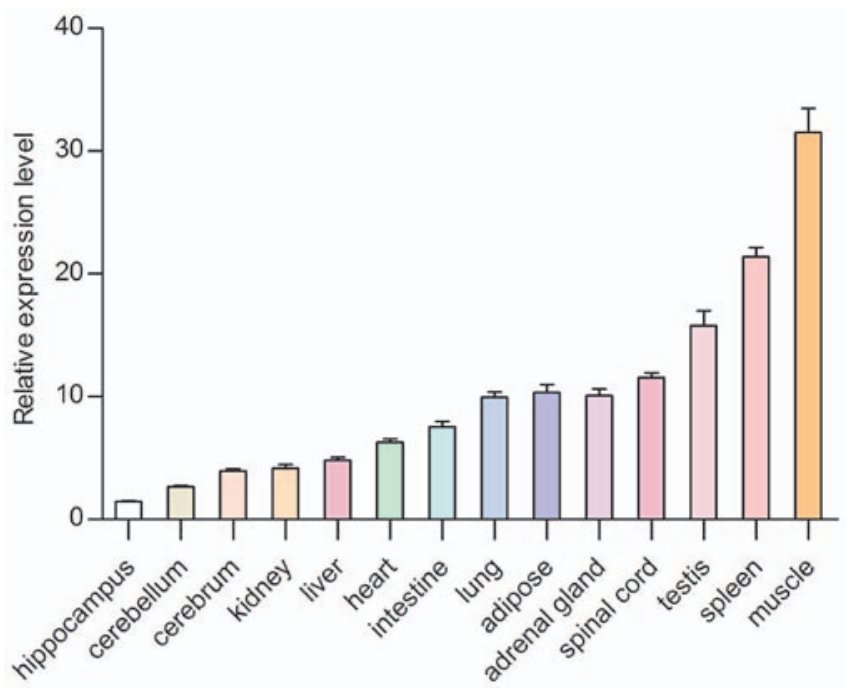

Figure 8. Analysis of mRNA expression profiles of tsIL-6. A single pair of primers for reverse transcription-quantitative polymerase chain reaction was designed to measure the mRNA expression of tsIL-6. The quantity in each sample was calculated according to its equation. tsIL-6, tress shrew interleukin-6.

post-injection with poly I:C, with the peak value of 24.3 -fold higher than that in the control at $12 \mathrm{~h}(\mathrm{P}<0.05)$, following which it reduced to the control level between 48 and $72 \mathrm{~h}$ (Fig. 10B).

A significant upregulation of tsIL- 6 was detected at $3 \mathrm{~h}$ post-LPS stimulation in the kidney $(\mathrm{P}<0.05)$, with athe peak value being 21.74-fold higher than that in the control, following which it returned to the control level between 12 and $72 \mathrm{~h}$ post-injection. The mRNA expression of tsIL-6 increased significantly following poly I:C challenge up to $6 \mathrm{~h}$ post-stimulation $(\mathrm{P}<0.05)$, with the peak value being 18.3 -fold higher than that in the control at $6 \mathrm{~h}(\mathrm{P}<0.05)$, following which it recovered to the control level between 12 and $72 \mathrm{~h}$ (Fig. 10C). A significant upregulation of tsIL-6 was detected in the intestinal tissues $3 \mathrm{~h}$ post-LPS stimulation $(\mathrm{P}<0.05)$, with the peak value being 25.5 -fold higher than that in the control, following which it returned to the control level between 6 and $72 \mathrm{~h}$ post-injection. The mRNA level of tsIL-6 increased significantly $6 \mathrm{~h}$ post poly I:C challenge $(\mathrm{P}<0.05)$, with the peak value being 19.66-fold higher than that in the control $(\mathrm{P}<0.05)$, followed by recovery to the control level between 12 and $72 \mathrm{~h}$ (Fig. 10D). 


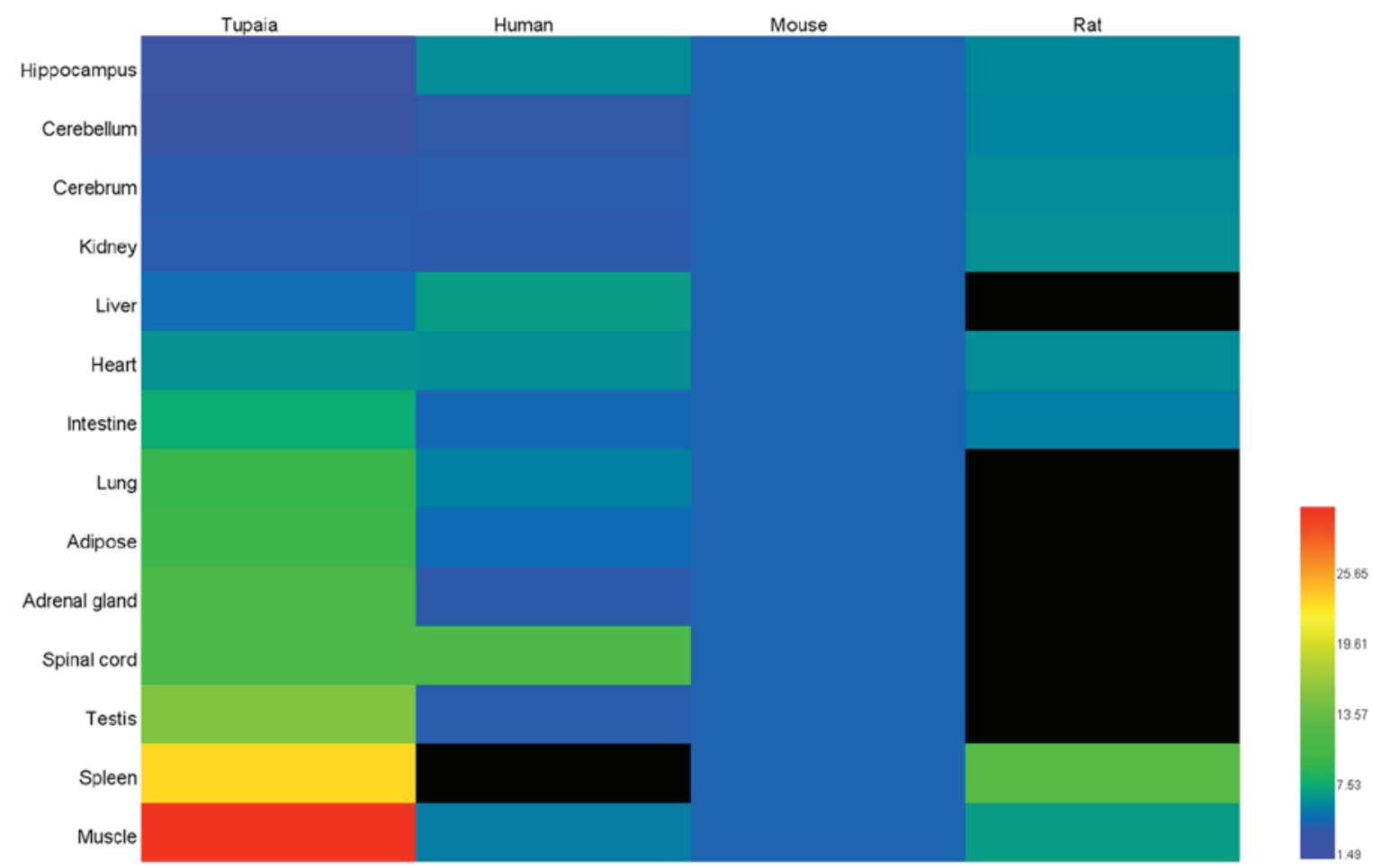

Figure 9. Heat map showing the basal gene expression of IL-6 in 14 tissues from tree shrew, human, mouse and rat. Human and mouse IL-6 gene expression data were obtained from BioGPS (www.biogps.org). Red-green heatmap represents gene expression levels in the 14 tissues (each row represents one tissue) and from tupaia, human, mouse and rat (each column represents one species). Black boxes indicated missing information in human or rat. The numbers represents the IL-6 relative expression level. IL-6, interleukin-6.

A

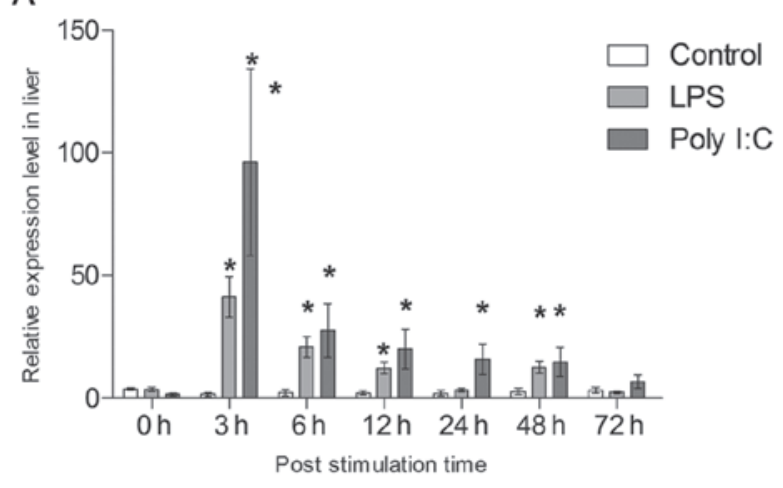

C

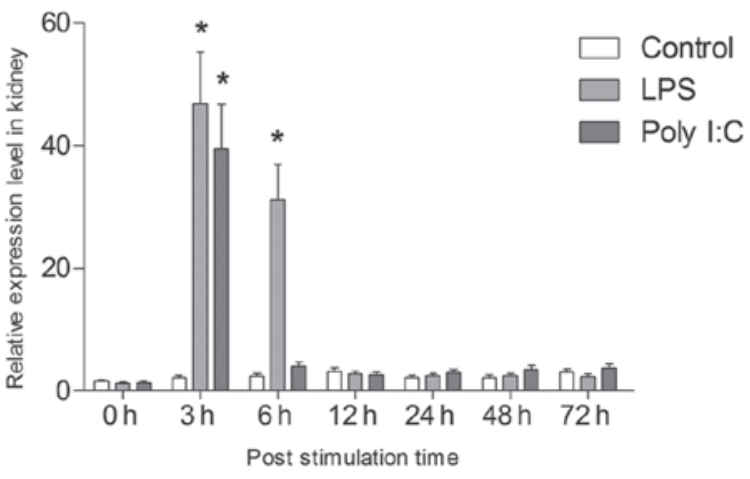

B

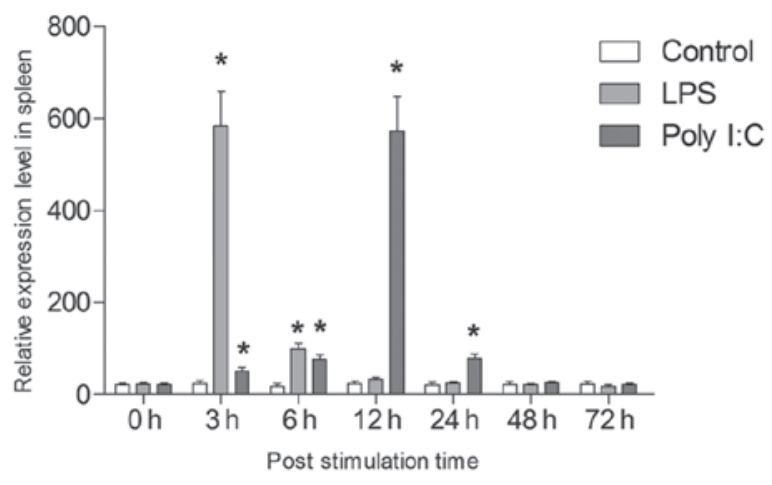

D

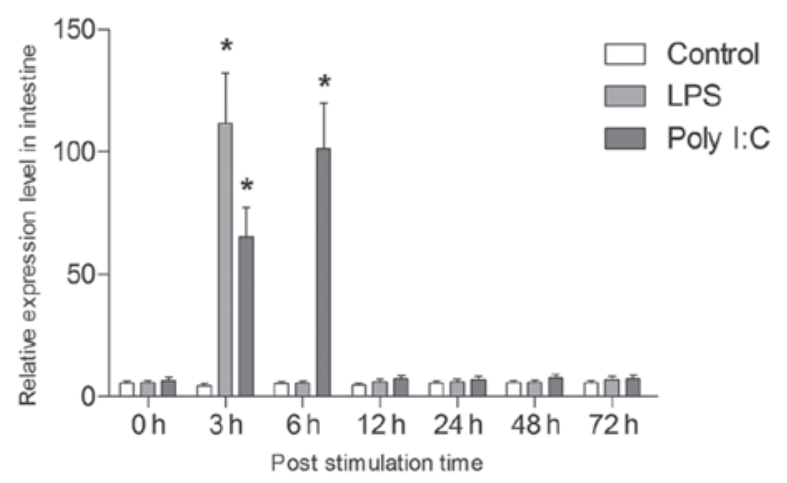

Figure 10. Analysis of the expression of tsIL-6. Expression of tsIL-6 was determined in the (A) liver, (B) spleen, (C) kidney and (D) intestinal tissues of the LPS- and poly I:C-challenged groups and the control group using reverse transcription-quantitative polymerase chain reaction analysis at $0,3,6,12,24,48$ and $72 \mathrm{~h}$-post injection. Data are presented as the mean \pm standard deviation $(\mathrm{n}=6)$ within the same post-injection time. ${ }^{*} \mathrm{P}<0.05$ among treatments. tsIL-6, tress shrew interleukin-6; LPS, lipopolysaccharide; poly I:C, polyinosinic:polycytidylic acid. 
Table III. Post-injection expression data.

Post-

injection

\begin{tabular}{|c|c|c|c|c|}
\hline Tissue & (h) & Control & LPS & Poly I:C \\
\hline \multirow[t]{7}{*}{ Liver } & 0 & $3.85 \pm 0.46$ & $3.70 \pm 0.73$ & $1.54 \pm 0.61$ \\
\hline & 3 & $1.55 \pm 0.82$ & $41.31 \pm 8.18$ & $96.13 \pm 38.07$ \\
\hline & 6 & $2.34 \pm 1.37$ & $20.78 \pm 4.11$ & $27.58 \pm 10.92$ \\
\hline & 12 & $2.20 \pm 0.94$ & $12.35 \pm 2.45$ & $20.00 \pm 7.92$ \\
\hline & 24 & $2.08 \pm 1.20$ & $3.50 \pm 0.69$ & $15.90 \pm 6.30$ \\
\hline & 48 & $2.78 \pm 1.37$ & $12.70 \pm 2.51$ & $14.71 \pm 5.82$ \\
\hline & 72 & $3.31 \pm 1.18$ & $2.50 \pm 0.49$ & $6.80 \pm 2.69$ \\
\hline \multirow[t]{7}{*}{ Spleen } & 0 & $22.14 \pm 4.07$ & $23.19 \pm 4.26$ & $22.03 \pm 4.05$ \\
\hline & 3 & $24.67 \pm 9.07$ & $583.57 \pm 107.29$ & $51.98 \pm 9.57$ \\
\hline & 6 & $19.13 \pm 7.03$ & $99.59 \pm 18.31$ & $76.12 \pm 13.99$ \\
\hline & 12 & $24.06 \pm 8.84$ & $33.45 \pm 6.15$ & $572.65 \pm 105.28$ \\
\hline & 24 & $21.33 \pm 7.83$ & $25.27 \pm 4.64$ & $78.10 \pm 14.36$ \\
\hline & 48 & $22.18 \pm 8.15$ & $21.56 \pm 3.96$ & $25.38 \pm 4.66$ \\
\hline & 72 & $23.00 \pm 8.46$ & $19.05 \pm 3.50$ & $22.28 \pm 4.11$ \\
\hline \multirow[t]{7}{*}{ Kidney } & 0 & $1.51 \pm 0.39$ & $1.25 \pm 0.32$ & $1.29 \pm 0.34$ \\
\hline & 3 & $2.15 \pm 0.56$ & $46.75 \pm 12.16$ & $39.42 \pm 10.25$ \\
\hline & 6 & $2.38 \pm 0.62$ & $31.20 \pm 8.11$ & $3.96 \pm 1.03$ \\
\hline & 12 & $3.14 \pm 0.82$ & $2.70 \pm 0.70$ & $2.58 \pm 0.67$ \\
\hline & 24 & $2.15 \pm 0.56$ & $2.40 \pm 0.62$ & $2.97 \pm 0.77$ \\
\hline & 48 & $2.17 \pm 0.57$ & $2.40 \pm 0.62$ & $3.51 \pm 0.91$ \\
\hline & 72 & $3.02 \pm 0.79$ & $2.30 \pm 0.60$ & $3.66 \pm 0.95$ \\
\hline \multirow[t]{7}{*}{ Intestine } & 0 & $5.36 \pm 1.39$ & $5.55 \pm 1.44$ & $6.56 \pm 1.71$ \\
\hline & 3 & $4.37 \pm 1.13$ & $111.65 \pm 29.03$ & $65.25 \pm 16.97$ \\
\hline & 6 & $5.15 \pm 1.34$ & $5.32 \pm 1.38$ & $101.25 \pm 26.33$ \\
\hline & 12 & $4.57 \pm 1.19$ & $5.98 \pm 1.55$ & $7.21 \pm 1.87$ \\
\hline & 24 & $5.32 \pm 1.38$ & $5.90 \pm 1.53$ & $6.90 \pm 1.79$ \\
\hline & 48 & $5.53 \pm 1.44$ & $5.65 \pm 1.47$ & $7.65 \pm 1.99$ \\
\hline & 72 & $5.52 \pm 1.44$ & $6.87 \pm 1.79$ & $7.32 \pm 1.90$ \\
\hline
\end{tabular}

Data are presented as the mean \pm standard deviation. LPS, lipopolysaccharide; poly I:C, polyinosinic:polycytidylic acid.

\section{Discussion}

IL-6-type cytokines are important in the communication between cells of multicellular organisms. They are involved in the regulation of complex cellular processes, including proliferation and differentiation, and are key during inflammation and the immune response. Elucidating the structures, functions and expression patterns of IL-6 is important for understanding their essential roles in normal tissue development and diseases. As tree shrews are increasingly used as novel disease animal models, it is important to understand the biology of tree shrews at the molecular level. However, IL-6 in the tree shrew has not been investigated previously. In the present study, full-length tsIL-6 genomic and mRNA sequences were first identified, and their evolutionary status was compared with other species. Subsequently, the conservation of their functional domains was analyzed, including signal peptides, conserved cysteine residues and typical IL-6/G-CSF/MGF family structure. In addition, the expression patterns in different tissues were analyzed prior to and following LPS and poly I:C challenge.

To date, three relevant sequences of tsIL- 6 have been recorded. One is in the 2 -fold coverage ( $2 \mathrm{X}$ ) genomic sequence assemblies of tree shrews, generated by Lindblad-Toh et al (25), and the other two are in the 70-fold coverage (70X) genomic sequence assemblies of tree shrews, generated by Fan et al (16). According to Lindblad-Toh et al (25), the partial mRNA of 570 bp encoding a 189-aa peptide has already been documented (ENSTBET00000004709) in the Ensembl database, although this incomplete. According to Fan et al (16), the partial mRNA of the 779-bp sequence encoding a 208-aa peptide has been previously predicted based on the shotgun sequencing method (Gene ID: 102496137) (1), which has not been experimentally identified.

As an important cytokine associated with the immune system, the present study identified the complete mRNA and genomic DNA sequence of tsIL-6 for the first time, and comprehensive analysis and characterization of tsIL- 6 was performed. The present study experimentally confirmed the presence of five exons and four introns in the tsIL- 6 gene, as previously predicted, and filled the genetic gap in the predicted genomic sequence. It was demonstrated that all four introns have a typical 'GU-AG' structure (Fig. 2).

In the present study, the full-length tsIL-6 mRNA was found to consist of $1,152 \mathrm{bp}$ with an ORF of $627 \mathrm{bp}$ encoding 208 amino acids. Six mRNA instability motifs (ATTTA), which are typical of genes encoding inflammatory mediators, were located in the tsIL-6 3'-UTR. These motifs suggested that this gene is transiently transcribed and involved in inflammatory responses. A 25-aa signal peptide and a conserved IL- 6 superfamily domain were predicted in the tsIL-6 protein, which agreed with the protein characterizations of IL-6 in humans (Homo sapiens). The majority of reported mammalian IL-6s contain four conserved cysteine residues, which are important for IL- 6 bioactivity. The tsIL-6 domain also contains the typical IL-6/G-CSF/MGF family structure of C-X(9)-C-X(6)-G-L-X(2)-[FY]-X(3)-L, indicating that tsIL-6 may share similar functions with the conservative IL-6 family.

The five exons identified from tsIL-6 genomic sequences were similar to the IL-6 structure in humans, monkeys and gorillas. Phylogenetic analysis demonstrated sister clades between tree shrews and the order of primates, with PP and BP support $(0.68 / 100)$. These results partly support the results from a previous study, with tree shrews constituting a sister clade to Primatomorpha (Dermoptera and Primates) other than Glires (rodents and lagomorphs) at the root of the Euarchontoglires (26).

Regarding amino acid length, mice and rats had missing amino acids, S and E, at positions 15 and 51, respectively, but an extra amino acid, $\mathrm{K}$, at position 137. Mice also had a missing 55-aa peptide tail in the $\mathrm{C}$ terminus. The tree shrew had three missing amino acids, SSK, at position 80 and a missing amino acid, S, at position 136. This suggested that position 136-137 is a mutation-prone site in IL-6. Regarding the amino acid sequence, human IL-6 had identities of 98.11, 96.70, 40.65, 30.05 and $52.36 \%$ with chimpanzee, monkey, rat, mouse and tree shrew IL-6, respectively. 
The homology modeling of the predicted tsIL-6 protein (Fig. 5) demonstrated a high level of structural homology with the crystallographic structure of human IL-6. Previous human IL-6 studies have demonstrated a hexameric structure and assembly of the IN-6/IL-6 $\alpha$-receptor/gp 130 complex (27). In the three primary IL- 6 binding sites, site I, II and III, the modification of amino acid Gln175 to Asn104 in site I; Gly35 to Glu35, Ser118 to Leu146, Val121 to Met149 in site II; and Asp160 to Lys184 in site III altered the surface charge (Fig. 6). The changes in these sites present an asymmetric pattern of evolution. The protein-protein interactions in human IL-6 and tsIL-6 may differ as a consequence of these changes.

The expression levels of IL- 6 in different tissues may indicate their functions in organ development, homeostasis and diseases. In the tree shrew, the present study determined the relative mRNA levels of IL-6 in 14 tissues from adult tree shrews. The highest expression level was found in muscle. Numerous studies have indicated that active skeletal muscle synthesizes and releases IL-6, which is important in the adaptation of an organism to exercise. Investigations in humans have also shown that human activity results in the production of IL-6 in muscles (28). Strenuous exercise can cause a marked increase in the plasma concentration of IL-6, which originates from skeletal muscle. The increase of IL-6 is relevant to the intensity and duration of exercise. As a result, muscles are considered to be the site of IL-6 production (29). Therefore, the high expression level of tsIL-6 may be associated with high locomotor abilities of tree shrew and tsIL- 6 may be important in skeletal muscle. The second highest expression level was found in the spleen, which is a tissue associated with the immune system. Moderate expression levels of IL-6 were observed in the testis, adipose tissue, spinal cord, adrenal gland, lung, intestine, heart, liver and kidney, whereas low expression levels were found in the cerebrum, cerebellum and hippocampus. The expression of tsIL- 6 was confirmed to exist widely in each examined tissue of the tree shrew by RT-qPCR analysis. The expression level in different tissues may be due to different species, immunological status, developmental stage or genetic background. Considering the living environment of tree shrews in the wild, the relatively high expression level of IL-6 may be associated with chronic infection, neoplasia and autoimmune diseases (30).

Previous studies have demonstrated that IL-6 transcripts can be induced by LPS or poly I:C stimulation in mice $(31,32)$, grouper (33) and human corneal fibroblasts (34). These studies indicated that IL- 6 transcripts were markedly induced under LPS and I:C stimulation in these models. Similarly, the results of the present study revealed that tsIL- 6 was induced in the liver, spleen, kidney and intestine following LPS and poly I:C stimulation, suggesting that IL- 6 can be induced in tree shrews as a defense against bacterial and viral infection.

In conclusion, cloning, sequencing and modeling of the tsIL-6 gene revealed molecular, structural and functional conservation between tree shrews and primates. The mRNA and genomic sequence of tsIL-6 generated in the present study offers an opportunity to elucidate the genetic basis of its suitability as an animal model for investigations associated with viral infections. The availability of these novel gene data provides a valuable basis and tool for functional gene investigations of tsIL-6.

\section{Acknowledgements}

This study was supported by Yunnan Science and Technology Talent and Platform Program (no. 2017HC019), the National Science and Technology Support Program (grant no. 2014BAI01B00), the Joint Support for the National Program of Yunnan Province (grant no. 2015GA009) and the National Nature Science Foundation of China (grant no. 31301044).

\section{References}

1. Yang EB, Cao J, Su JJ and Chow P: The tree shrews: Useful animal models for the viral hepatitis and hepatocellular carcinoma. Hepatogastroenterology 52: 613-616, 2005.

2. Fuchs E: Social stress in tree shrews as an animal model of depression: An example of a behavioral model of a CNS disorder. CNS 10: 182-190, 2005.

3. Norton TT and Siegwart JT Jr: Light levels, refractive development, and myopia - a speculative review. Exp Eye Res 114: 48-57, 2013.

4. Guo L, Frost MR, Siegwart JT Jr and Norton TT: Scleral gene expression during recovery from myopia compared with expression during myopia development in tree shrew. Mol Vis 20: 1643-1659, 2014.

5. Ye L, He M, Huang Y, Zhao G, Lei Y, Zhou Y and Chen X: Tree shrew as a new animal model for the study of lung cancer. Oncol Lett 11: 2091-2095, 2016.

6. Lin N, Xiong LL, Zhang RP, Zheng H, Wang L, Qian ZY, Zhang P, Chen ZW, Gao FB and Wang TH: Injection of A $\beta 1-40$ into hippocampus induced cognitive lesion associated with neuronal apoptosis and multiple gene expressions in the tree shrew. Apoptosis 21: 621-640, 2016.

7. Hunter CA and Jones SA: IL-6 as a keystone cytokine in health and disease. Nat Immunol 16: 448-457, 2015.

8. Lohrengel B, Lu M and Roggendorf M: Molecular cloning of the woodchuck cytokines: TNF-alpha, IFN-gamma, and IL-6. Immunogenetics 47: 332-335, 1998.

9. Nagarajan G, Swami SK, Ghorui SK, Pathak KM, Singh RK and Patil NV: Cloning and phylogenetic analysis of interleukin-6 (IL-6) and tumor necrosis factor- $\alpha$ (TNF- $\alpha$ ) from Indian dromedaries (Camelus dromedarius). Comp Immunol Microbiol Infect Dis 34: 291-298, 2011.

10. Takakura H, Mori Y and Tatsumi M: Molecular cloning of caprine IL-6 cDNA and its expression in insect cells. Int Arch Allergy Immunol 113: 409-416, 1997.

11. Zhu Q, Li C, Yu ZX, Zou PF, Meng QX and Yao CL: Molecular and immune response characterizations of IL-6 in large yellow croaker (Larimichthys crocea). Fish Shellfish Immunol 50: 263-273, 2016.

12. Iliev DB, Castellana B, Mackenzie S, Planas JV and Goetz FW: Cloning and expression analysis of an IL-6 homolog in rainbow trout (Oncorhynchus mykiss). Mol Immunol 44: 1803-1807, 2007.

13. Yu D, Wu Y, Xu L, Fan Y, Peng L, Xu M and Yao YG: Identification and characterization of toll-like receptors (TLRs) in the Chinese tree shrew (Tupaia belangeri chinensis). Dev Comp Immunol 60: 127-138, 2016.

14. Yu D, Xu L, Liu XH, Fan Y, Lü LB and Yao YG: Diverse interleukin-7 mRNA transcripts in Chinese tree shrew (Tupaia belangeri chinensis). PLoS One 9: e99859, 2014.

15. Zheng Y, Yun C, Wang Q, Smith WW and Leng J: Identification of the full-length $\beta$-actin sequence and expression profiles in the tree shrew (Tupaia belangeri). Int J Mol Med 35: 519-524, 2015.

16. Fan Y, Yu D and Yao YG: Tree shrew database (TreeshrewDB): A genomic knowledge base for the Chinese tree shrew. Sci Rep 4: 7145, 2014.

17. Jain R, Gomer RH and Murtagh JJ Jr: Increasing specificity from the PCR-RACE technique. Biotechniques 12: 58-59, 1992.

18. Edgar RC: MUSCLE: Multiple sequence alignment with high accuracy and high throughput. Nucleic Acids Res 32: 1792-1797, 2004.

19. Ronquist F and Huelsenbeck JP: MrBayes 3: Bayesian phylogenetic inference under mixed models. Bioinformatics 19: 1572-1574, 2003.

20. Meyer X, Chopard B and Salamin N: Accelerating Bayesian inference for evolutionary biology models. Bioinformatics 33: 669-676, 2017. 
21. Stamatakis A, Ludwig T and Meier H: RAxML-III: A fast program for maximum likelihood-based inference of large phylogenetic trees. Bioinformatics 21: 456-463, 2005

22. Remans T, Smeets K, Opdenakker K, Mathijsen D, Vangronsveld J and Cuypers A: Normalisation of real-time RT-PCR gene expression measurements in Arabidopsis thaliana exposed to increased metal concentrations. Planta 227: 1343-1349, 2008.

23. Siemetzki U, Ashok MS, Briese T and Lipkin WI: Identification of RNA instability elements in Borna disease virus. Virus Res 144: 27-34, 2009.

24. Fu X, Ding Z, Fan J, Wang H, Zhou F, Cui L, Boxiang C, Wang W and Liu H: Characterization, promoter analysis and expression of the interleukin-6 gene in blunt snout bream, Megalobrama amblycephala. Fish Physiol Biochem 42: 1527-1540, 2016.

25. Lindblad-Toh K, Garber M,Zuk O, Lin MF, Parker BJ, Washietl S, Kheradpour P, Ernst J, Jordan G, Mauceli E, et al; Broad Institute Sequencing Platform and Whole Genome Assembly Team; Baylor College of Medicine Human Genome Sequencing Center Sequencing Team; Genome Institute at Washington University: A high-resolution map of human evolutionary constraint using 29 mammals. Nature 478: 476-482, 2011.

26. Zhou X, Sun F, Xu S, Yang G and Li M: The position of tree shrews in the mammalian tree: Comparing multi-gene analyses with phylogenomic results leaves monophyly of Euarchonta doubtful. Integr Zool 10: 186-198, 2015.

27. Boulanger MJ, Chow DC, Brevnova EE and Garcia KC: Hexameric structure and assembly of the interleukin-6/IL-6 alpha-receptor/gp130 complex. Science 300: 2101-2104, 2003.

28. Ikeda SI, Tamura Y, Kakehi S, Sanada H, Kawamori R and Watada H: Exercise-induced increase in IL-6 level enhances GLUT4 expression and insulin sensitivity in mouse skeletal muscle. Biochem Biophys Res Commun 473: 947-952, 2016.
29. Ono T, Maekawa K, Watanabe S, Oka H and Kuboki T: Muscle contraction accelerates IL- 6 mRNA expression in the rat masseter muscle. Arch Oral Biol 52: 479-486, 2007.

30. Gleeson M: Interleukins and exercise. J Physiol 529: 1, 2000.

31. Hacham M, Cristal N, White RM, Segal S and Apte RN: Complementary organ expression of IL-1 vs. IL-6 and CSF-1 activities in normal and LPS-injected mice. Cytokine 8: 21-31, 1996.

32. Szot P, Franklin A, Figlewicz DP, Beuca TP, Bullock K, Hansen K, Banks WA, Raskind MA and Peskind ER: Multiple lipopolysaccharide (LPS) injections alter interleukin 6 (IL-6), IL-7, IL-10 and IL-6 and IL-7 receptor mRNA in CNS and spleen. Neuroscience 355: 9-21, 2017.

33. Jeong YH, Park JS, Kim DH, Kang JL and Kim HS: Antiinflammatory mechanism of lonchocarpine in LPS- or poly(I:C)-induced neuroinflammation. Pharmacol Res 119: 431-442, 2017.

34. Kimura K, Orita T, Nomi N, Fujitsu Y, Nishida T and Sonoda KH: Identification of common secreted factors in human corneal fibroblasts exposed to LPS, poly(I:C), or zymosan. Exp Eye Res 96: 157-162, 2012.

(i) (9) This work is licensed under a Creative Commons Attribution-NonCommercial-NoDerivatives 4.0 International (CC BY-NC-ND 4.0) License. 